\title{
SUPERSYMMETRY OF FRW BAROTROPIC COSMOLOGIES
}

\author{
H.C. ROSU* and P. OJEDA-MAY ${ }^{\dagger}$ \\ Potosinian Institute of Science and Technology (IPICyT) \\ Apartado Postal 3-74 Tangamanga, 78231 San Luis Potosí, Mexico
}

\begin{abstract}
Barotropic FRW cosmologies are presented from the standpoint of nonrelativistic supersymmetry. First, we reduce the barotropic FRW system of differential equations to simple harmonic oscillator differential equations. Employing the factorization procedure, the solutions of the latter equations are divided into the two classes of bosonic (nonsingular) and fermionic (singular) cosmological solutions. We next introduce a coupling parameter denoted by $K$ between the two classes of solutions and obtain barotropic cosmologies with dissipative features acting on the scale factors and spatial curvature of the universe. The K-extended FRW equations in comoving time are presented in explicit form in the low coupling regime. The standard barotropic FRW cosmologies correspond to the dissipationless limit $\mathrm{K}=0$.
\end{abstract}

\section{Comoving FRW barotropy}

Barotropic FRW cosmologies in comoving time t obey the Einstein-Friedmann dynamical equations for the scale factor $\mathrm{a}(\mathrm{t})$ of the universe supplemented by the (barotropic) equation of state of the cosmological fluid

$$
\begin{aligned}
& \frac{\ddot{a}}{\mathrm{a}}=-\frac{4 \pi \mathrm{G}}{3}(\rho+3 \mathrm{p}), \\
& \mathrm{H}_{0}^{2}(\mathrm{t}) \equiv\left(\frac{\dot{a}}{\mathrm{a}}\right)^{2}=\frac{8 \pi \mathrm{G} \rho}{3}-\frac{\kappa}{\mathrm{a}^{2}}, \\
& \mathrm{p}=(\gamma-1) \rho,
\end{aligned}
$$

where $\rho$ and $\mathrm{p}$ are the energy density and the pressure, respectively, of the perfect fluid of which a classical universe is usually assumed to be made of, $\kappa=0, \pm 1$ is the curvature index of the flat, closed, open universe, respectively, and $\gamma$ is the constant adiabatic index of the cosmological fluid.

*E-mail: hcr@ipicyt.edu.mx

$\mathrm{h} \operatorname{crcos} 1$. tex

${ }^{\dagger}$ E-mail: pedro@ipicyt.edu.mx 
$\underline{\text { Bosonic FRW barotropy }}$

Passing to the conformal time variable $\eta$, defined through $\mathrm{dt}=\mathrm{a}(\eta) \mathrm{d} \eta$, one can combine the three equations in a single Riccati equation for the Hubble parameter $\mathrm{H}_{0}(\eta)$ (we shall use either $\frac{\mathrm{d}}{\mathrm{d} \eta}$ or ' for the derivative with respect to $\eta$ in the following)

$$
\mathrm{H}_{0}^{\prime}+\mathrm{cH}_{0}^{2}+\kappa \mathrm{c}=0
$$

where $\mathrm{c}=\frac{3}{2} \gamma-1$.

Employing now $\mathrm{H}_{0}(\eta)=\frac{1}{\mathrm{c}} \frac{\mathrm{w}^{\prime}}{\mathrm{w}}$ one gets the very simple (harmonic oscillator) second order differential equation

$$
\mathrm{w}^{\prime \prime}-\mathrm{c} \cdot \mathrm{c}_{\kappa, \mathrm{b}} \mathrm{w}=0
$$

where $\mathrm{c}_{\kappa, \mathrm{b}}=-\kappa \mathrm{c}$. Moreover, the particular Riccati solutions $\mathrm{H}_{0}^{+}=-\tan \mathrm{c} \eta$ and $\mathrm{H}_{0}^{-}=\operatorname{coth} \mathrm{c} \eta$ for $\kappa= \pm 1$, respectively, are related to the common factorizations of the equation (2)

$$
\left(\frac{\mathrm{d}}{\mathrm{d} \eta}+\mathrm{cH}_{0}\right)\left(\frac{\mathrm{d}}{\mathrm{d} \eta}-\mathrm{cH}_{0}\right) \mathrm{w}=\mathrm{w}^{\prime \prime}-\mathrm{c}\left(\mathrm{H}_{0}^{\prime}+\mathrm{cH}_{0}^{2}\right) \mathrm{w}=0 .
$$

Borrowing a terminology from supersymmetric quantum mechanics, we call the solutions $\mathrm{w}$ as bosonic zero modes. They are the following. For $\kappa=1$

$$
\mathrm{w}_{1, \mathrm{~b}} \sim \cos (\mathrm{c} \eta+\mathrm{d}) \quad \rightarrow \quad \mathrm{a}_{1, \mathrm{~b}}(\eta) \sim \mathrm{w}_{1}^{1 / \mathrm{c}}
$$

where $\mathrm{d}$ is an arbitrary phase, whereas for $\kappa=-1$ one gets

$$
\mathrm{w}_{-1, \mathrm{~b}} \sim \sinh (\mathrm{c} \eta) \quad \rightarrow \quad \mathrm{a}_{-1, \mathrm{~b}}(\eta) \sim \mathrm{w}_{-1}^{1 / \mathrm{c}} .
$$

\section{Fermionic FRW barotropy}

A class of barotropic FRW cosmologies with inverse scale factors with respect to the bosonic ones can be obtained by considering the supersymmetric partner (or fermionic) equation of Eq. (3) which is obtained by applying the factorization brackets in reverse order

$$
\left(\frac{\mathrm{d}}{\mathrm{d} \eta}-\mathrm{cH}_{0}\right)\left(\frac{\mathrm{d}}{\mathrm{d} \eta}+\mathrm{cH}_{0}\right) \mathrm{w}=\mathrm{w}^{\prime \prime}-\mathrm{c}\left(-\mathrm{H}_{0}^{\prime}+\mathrm{cH}_{0}^{2}\right) \mathrm{w}=0 .
$$

Thus, one can write

$$
\mathrm{w}^{\prime \prime}-\mathrm{c} \cdot \mathrm{c}_{\kappa, \mathrm{f}} \mathrm{w}=0
$$

where

$$
\mathrm{c}_{\kappa, \mathrm{f}}(\eta)=-\mathrm{H}_{0}^{\prime}+\mathrm{cH}_{0}^{2}= \begin{cases}c\left(1+2 \tan ^{2} c \eta\right) & \text { if } \kappa=1 \\ c\left(-1+2 \operatorname{coth}^{2} c \eta\right) & \text { if } \kappa=-1\end{cases}
$$


denotes the supersymmetric partner adiabatic index of fermionic type associated through the mathematical scheme to the constant bosonic index. Notice that the fermionic adiabatic index is time dependent. The fermionic w solutions are

$$
\mathrm{w}_{1, \mathrm{f}}=\frac{\mathrm{c}}{\cos (\mathrm{c} \eta+\mathrm{d})} \quad \rightarrow \quad \mathrm{a}_{1, \mathrm{f}}(\eta) \sim[\cos (\mathrm{c} \eta+\mathrm{d})]^{-1 / \mathrm{c}}
$$

and

$$
\mathrm{w}_{-1, \mathrm{f}}=\frac{\mathrm{c}}{\sinh (\mathrm{c} \eta)} \quad \rightarrow \quad \mathrm{a}_{-1, \mathrm{f}}(\eta) \sim[\sinh (\mathrm{c} \eta)]^{-1 / \mathrm{c}}
$$

for $\kappa=1$ and $\kappa=-1$, respectively.

We can see that the bosonic and fermionic barotropic cosmologies are reciprocal to each other, in the sense that

$$
\mathrm{a}_{ \pm, \mathrm{b}} \mathrm{a}_{ \pm, \mathrm{f}}=\text { const }
$$

Thus, bosonic expansion corresponds to fermionic contraction and viceversa.

\section{Uncoupled fermionic and bosonic FRW barotropies}

A matrix formulation of the previous results is possible as follows. Introducing the following two Pauli matrices

$$
\alpha=-\mathrm{i} \sigma_{\mathrm{y}}=-\mathrm{i}\left(\begin{array}{cc}
0 & -\mathrm{i} \\
\mathrm{i} & 0
\end{array}\right) \quad \text { and } \quad \beta=\sigma_{\mathrm{x}}=\left(\begin{array}{cc}
0 & 1 \\
1 & 0
\end{array}\right)
$$

we can write a cosmological matrix equation

$$
\sigma_{\mathrm{y}} \mathrm{D}_{\eta} \mathrm{W}+\sigma_{\mathrm{x}}\left(\mathrm{icH}_{0}\right) \mathrm{W}=0
$$

where $\mathrm{W}=\left(\begin{array}{c}w_{1} \\ w_{2}\end{array}\right)$ is a two component 'zero-mass' spinor. This is equivalent to the following decoupled equations

$$
\begin{array}{r}
\mathrm{D}_{\eta} w_{1}+\mathrm{cH}_{0} w_{1}=0 \\
-\mathrm{D}_{\eta} w_{2}+\mathrm{cH}_{0} w_{2}=0 .
\end{array}
$$

Solving these equations one gets $w_{1} \propto 1 / \cos (\mathrm{c} \eta)$ and $w_{2} \propto \cos (\mathrm{c} \eta)$ for $\kappa=1$ cosmologies and $w_{1} \propto 1 / \sinh (\mathrm{c} \eta)$ and $w_{2} \propto \sinh (\mathrm{c} \eta)$ for $\kappa=-1$ cosmologies. Thus, we obtain

$$
W=\left(\begin{array}{c}
w_{1} \\
w_{2}
\end{array}\right)=\left(\begin{array}{c}
\mathrm{w}_{\mathrm{f}} \\
\mathrm{w}_{\mathrm{b}}
\end{array}\right)
$$

This shows that the matrix equation contains the two reciprocal barotropic cosmologies on the same footing as the two components of the spinor $\mathrm{W}$. 
Coupled fermionic and bosonic cosmological barotropies

There is a simple way to couple the two spinorial components by means of a constant parameter $\mathrm{K}$. Indeed, we write

$$
\left[\sigma_{\mathrm{y}} \mathrm{D}_{\eta}+\sigma_{\mathrm{x}}\left(\mathrm{icH}_{0}+\mathrm{K}\right)\right] W=\mathrm{K} W
$$

where $\mathrm{K}$ is equivalent to the mass parameter of a Dirac spinor. Eq. (9) is equivalent to the following system of coupled equations

$$
\begin{array}{r}
\mathrm{D}_{\eta} \mathrm{w}_{1}+\left(\mathrm{icH}_{0}+\mathrm{K}\right) \mathrm{w}_{1}=\mathrm{Kw}_{2} \\
-\mathrm{D}_{\eta} \mathrm{w}_{2}+\left(\mathrm{icH}_{0}+\mathrm{K}\right) \mathrm{w}_{2}=\mathrm{Kw}_{1} .
\end{array}
$$

These two coupled first-order equations are equivalent to second order differential equations for each of the two spinor components.

The fermionic spinor component can be found directly as solutions of

$$
\begin{cases}\mathrm{w}_{1}^{+^{\prime \prime}}-\mathrm{c}\left[\mathrm{c}_{1, \mathrm{f}}(\eta)+2 \mathrm{iK} \tan \mathrm{c} \eta\right] \mathrm{w}_{1}^{+}=0 & \text { for } \kappa=1 \\ \mathrm{w}_{1}^{-{ }^{\prime \prime}}-\mathrm{c}\left[\mathrm{c}_{-1, \mathrm{f}}(\eta)-2 \mathrm{iK} \operatorname{coth} \mathrm{c} \eta\right] \mathrm{w}_{1}^{-}=0 & \text { for } \kappa=-1,\end{cases}
$$

whereas the bosonic components are solutions of

$$
\begin{cases}\mathrm{w}_{2}^{+\prime \prime}+c[c-2 \mathrm{iK} \tan \mathrm{c} \eta] \mathrm{w}_{2}^{+}=0 & \text { for } \quad \kappa=1 \\ \mathrm{w}_{2}^{-\prime \prime}+\mathrm{c}[-\mathrm{c}+2 \mathrm{iK} \operatorname{Kith} \mathrm{c} \eta] \mathrm{w}_{2}^{-}=0 & \text { for } \quad \kappa=-1 .\end{cases}
$$

The solutions of the bosonic equations are expressed in terms of the Gauss hypergeometric functions ${ }_{2} \mathrm{~F}_{1}$ of complex parameters that can be written in explicit form :

$$
\begin{array}{r}
\mathrm{z}_{2}^{-\mathrm{k}_{2}} \mathrm{w}_{2}^{+}(\eta)=\mathcal{A} \mathrm{z}_{1}^{\mathrm{k}_{1}}{ }_{2} \mathrm{~F}_{1}\left[\mathrm{k}_{1}+\mathrm{k}_{2}+1, \mathrm{k}_{1}+\mathrm{k}_{2}, 1+2 \mathrm{k}_{1} ;-\frac{\mathrm{z}_{1}}{2}\right] \\
-\mathcal{B} \mathrm{e}^{-\mathrm{i}\left(1+2 \mathrm{k}_{1}\right) \pi}\left(\frac{4}{\mathrm{z}_{1}}\right)^{\mathrm{k}_{1}}{ }_{2} \mathrm{~F}_{1}\left[-\mathrm{k}_{1}+\mathrm{k}_{2},-\mathrm{k}_{1}+\mathrm{k}_{2}+1,1-2 \mathrm{k}_{1} ;-\frac{\mathrm{z}_{1}}{2}\right]
\end{array}
$$

and

$$
\begin{gathered}
\mathrm{z}_{4}^{-\mathrm{k}_{4}} \mathrm{w}_{2}^{-}(\eta)=\mathcal{C} \mathrm{z}_{3}^{\mathrm{k}_{3}}{ }_{2} \mathrm{~F}_{1}\left[\mathrm{k}_{3}+\mathrm{k}_{4}, \mathrm{k}_{3}+\mathrm{k}_{4}+1,1+2 \mathrm{k}_{3} ; \frac{\mathrm{z}_{3}}{2}\right] \\
+\mathcal{D}\left(\frac{4}{\mathrm{z}_{3}}\right)^{\mathrm{k}_{3}}{ }_{2} \mathrm{~F}_{1}\left[-\mathrm{k}_{3}+\mathrm{k}_{4}+1,-\mathrm{k}_{3}+\mathrm{k}_{4}, 1-2 \mathrm{k}_{3} ; \frac{\mathrm{z}_{3}}{2}\right],
\end{gathered}
$$

where the variables $\mathrm{z}_{i}(i=1, \ldots, 4)$ are given in the following form:

$\mathrm{z}_{1}=\mathrm{i} \tan (\mathrm{c} \eta)-1, \quad \mathrm{z}_{2}=\mathrm{i} \tan (\mathrm{c} \eta)+1, \quad \mathrm{z}_{3}=\operatorname{coth}(\mathrm{c} \eta)+1, \quad \mathrm{z}_{4}=\operatorname{coth}(\mathrm{c} \eta)-1$, 
respectively. The $\mathrm{k}$ parameters are the following:

$$
\mathrm{k}_{1}=\frac{1}{2}\left(1-\frac{2 \mathrm{~K}}{\mathrm{c}}\right)^{\frac{1}{2}}, \quad \mathrm{k}_{2}=\frac{1}{2}\left(1+\frac{2 \mathrm{~K}}{\mathrm{c}}\right)^{\frac{1}{2}}
$$

and

$$
\mathrm{k}_{3}=\frac{1}{2}\left(1+\mathrm{i} \frac{2 \mathrm{~K}}{\mathrm{c}}\right)^{\frac{1}{2}}, \quad \mathrm{k}_{4}=\frac{1}{2}\left(1-\mathrm{i} \frac{2 \mathrm{~K}}{\mathrm{c}}\right)^{\frac{1}{2}},
$$

whereas $\mathcal{A}, \mathcal{B}, \mathcal{C}, \mathcal{D}$ are superposition constants. Plots of these modes are given in Ref. $2 \mathrm{~b}$.

Based on these $\mathrm{K}$ zero-modes, we can introduce bosonic scale factors and Hubble parameters depending on the parameter $\mathrm{K}$

$$
\mathrm{a}_{\mathrm{K},+}=\left(\mathrm{w}_{2}^{+}\right)^{1 / \mathrm{c}}, \quad \mathrm{H}_{\mathrm{K}}^{+}(\eta)=\frac{1}{\mathrm{c}}\left(\log \mathrm{w}_{2}^{+}\right)^{\prime}
$$

and

$$
\mathrm{a}_{\mathrm{K},-}=\left(\mathrm{w}_{2}^{-}\right)^{1 / \mathrm{c}}, \quad \mathrm{H}_{\mathrm{K}}^{-}(\eta)=\frac{1}{\mathrm{c}}\left(\log \mathrm{w}_{2}^{-}\right)^{\prime},
$$

and similarly for the fermionic components by changing $\mathrm{w}_{2}^{ \pm}$to $\mathrm{w}_{1}^{ \pm}$in eqs. (16) and (17), respectively.

\section{Comoving K-coupled FRW barotropy: Small K regime}

Introducing the notations $\lambda_{\mathrm{K}}=-\mathrm{K}\left(\frac{\partial \mathrm{a}_{\mathrm{K}}}{\partial \mathrm{K}}\right)_{\mathrm{K}=0}$ and $\mathrm{F}_{\kappa}(\mathrm{t})=\left(1+\frac{2 \lambda_{\mathrm{K}}}{\kappa \mathrm{a}_{\mathrm{K}}}\right)$, one can show that in the small $\mathrm{K} / \mathrm{c}$ limit the comoving time equations can be written as follows:

$$
\begin{aligned}
& \frac{\ddot{a}_{\mathrm{K}}}{\mathrm{a}_{\mathrm{K}}}\left(\mathrm{F}_{1}(\mathrm{t})-\frac{\lambda_{\mathrm{K}}}{\mathrm{a}_{\mathrm{K}}}\right)-\frac{\ddot{\lambda}_{\mathrm{K}}}{\mathrm{a}_{\mathrm{K}}}=-\frac{4 \pi \mathrm{G}}{3}(\rho+3 \mathrm{p}), \\
& \left(\frac{\dot{\mathrm{a}}_{\mathrm{K}}}{\mathrm{a}_{\mathrm{K}}}\right)^{2} \mathrm{~F}_{1}(\mathrm{t})=\frac{8 \pi \mathrm{G} \rho}{3}-\frac{\left(\kappa+2 \frac{\lambda_{\mathrm{K}}}{\mathrm{a}_{K}}\right)}{\mathrm{a}_{\mathrm{K}}^{2}}, \\
& \mathrm{p}=(\gamma-1) \rho .
\end{aligned}
$$

( $a_{K}$ could be either $a_{K,+}$ or $a_{K,-}$ depending on the $\kappa$ case we take into account).

\section{Interpretation}

We come now to the interpretational issue. We consider only the small $\mathrm{K}$ regime as realistic. Then, the effects of $\mathrm{K}$ show up only on the geometrical quantities without any change in the barotropic equation of state. The parameter $\mathrm{K}$ introduces an imaginary part in the cosmological Hubble parameter $\mathrm{H}$. Since the latter is the logarithmic derivative of the scale factor of the universe one comes to the conclusion that the supersymmetric techniques presented here are a supersymmetric way to take into account dissipation 
and instabilities of barotropic FRW cosmologies. However, since $\mathrm{K}$ is also a coupling parameter between fermion and boson components, the dissipation and instabilities belong to the cosmological epochs that occurred before the supersymmetry breaking.

\section{References}

1. V. Faraoni, "Solving for the dynamics of the universe", Am. J. Phys. 67, 732 (1999), physics/9901006

2. a) H.C. Rosu, "Darboux class of cosmological fluids with time-dependent adiabatic indices", Mod. Phys. Lett. A 15, 979 (2000), gr-qc/0003108

b) H.C. Rosu, O. Cornejo-Pérez, R. López-Sandoval, "Classical harmonic oscillator with Dirac-like parameters and possible applications", J. Phys. A 37, 11699 (2004), math-ph/0402065

c) H.C. Rosu and R. López-Sandoval, "Barotropic FRW cosmologies with a Dirac-like parameter", Mod. Phys. Lett. A 19, 1529 (2004), gr-qc/0403045

3. a) F. Cooper, A. Khare, R. Musto, A. Wipf, "Supersymmetry and the Dirac equation", Ann. Phys. 187, 1 (1988). See also:

b) R.J. Hughes, V. Alan Kostelecký, M.M. Nieto, "Susy quantum mechanics in a firstorder Dirac equation", Phys. Rev. D 34, 1100 (1986);

c) Y. Nogami and F.M. Toyama, "Supersymmetry aspects of the Dirac equation in one dimension with a Lorentz scalar potential", Phys. Rev. A 47, 1708 (1993). 